\section{Post-surgical pain, physical activity and satisfaction with the decision to undergo hernia surgery: a prospective qualitative investigation}

\author{
Rachael Powell, 1 Lorna McKee, 2 \\ Peter M. King,3 Julie Bruce4
}

1School of Psychological Sciences, University of Manchester, Manchester; 2Health Services Research Unit, University of Aberdeen, Aberdeen; 3Department of Surgery, Aberdeen Royal Infirmary, Aberdeen; ${ }^{4}$ Warwick Clinical Trials Unit, University of Warwick, Coventry, UK

\section{Abstract}

Surgical repair is a common treatment for inguinal hernias but a substantial number of patients experience chronic pain after surgery. As some patients are pain-free on presentation, it is important to investigate whether patients perceive the treatment to be beneficial. The present study used qualitative methods to explore experiences of pain, activity limitations and satisfaction with treatment as people underwent surgery and recovery. Twenty-nine semi-structured interviews were conducted. Seven participants were interviewed longitudinally: before surgery and two weeks and four months post-surgery. Ten further participants with residual pain four months post-surgery were interviewed once. Semi-structured interviews included experience and perception of pain; activity limitations; reasons for having surgery; satisfaction with the decision to undergo surgery. A thematic analysis was conducted. Pain did not cause concern when perceived as part of the usual surgery and recovery processes. Activity was limited to avoid damage to the hernia site rather than to avoid pain. None of the participants reported dissatisfaction with the decision to have surgery; reducing the risk of lifethreatening complications associated with untreated hernias was considered important. These findings suggest that people regarded surgical treatment as worthwhile, despite chronic post-surgical pain. Further research should ascertain whether patients are aware of the actual risk of complications associated with conservative rather than surgical management of inguinal hernia.

\section{Introduction}

Surgery is an effective treatment for people with inguinal hernia, a common condition with an incidence of $6-12 \%$ in adult males and approximately a tenth of this in females. ${ }^{1}$ It is a routine, elective procedure yet a considerable proportion of patients experience chronic post-surgical pain (CPSP). CPSP is pain that persists beyond the normal healing time, usually taken as 3 months post-surgery. ${ }^{2}$ In the context of CPSP after hernia surgery, this pain is experienced in the locality of the hernia surgery. In a review of 40 studies, the frequency of CPSP after hernia surgery ranged from 0 to $54 \% .^{3}$ Bay-Nielsen et al. found $29 \%$ of participants reported pain 1 year post-surgery; over half of those with CPSP also reported restriction in activities due to the pain. ${ }^{4}$ In a retrospective study, $20 \%$ of those with CPSP after inguinal hernia repair reported that they would not have undergone surgery had they understood the risk of CPSP. ${ }^{5}$ Some people have only minor symptoms before surgery so it is important to understand why they decide to undergo surgery and to explore satisfaction with this choice when CPSP is experienced.

An inguinal hernia usually presents as a lump in the groin (fat or other intra-abdominal viscera protruding through the abdominal wall); patients may experience pain. Occasionally the condition becomes life-threatening when the protrusion strangulates (insufficient blood supply) or the intestine becomes obstructed. ${ }^{6}$ Surgery is performed to alleviate discomfort and prevent dangerous complications which require emergency surgery.

Hair et al. found that $66 \%$ of people presenting with inguinal hernias reported pain; ${ }^{7}$ the likelihood of pain increased in those who had had the hernia for longer. One third, therefore, were pain-free on presentation. This raises the question of whether those with little or no pain would prefer a watchful waiting strategy over a surgical procedure that could result in CPSP.

It is not clear whether the risk of complications is such that surgery is preferable to watchful waiting given CPSP risk. Leubner et al. reported the strangulation risk to be less than $1 \%$ per year in the first few years after onset. ${ }^{8}$ Two randomised controlled trials compared watchful waiting with surgical repair in asymptomatic males. Fitzgibbons et al. ${ }^{9}$ enrolled adults aged $\geq 18$ years with follow-up for two years; 0'Dwyer et al.'s trial included adults aged $\geq 55$ years and followed them to one year after recruitment. ${ }^{10}$ Both studies found no difference in pain ratings between watchful waiting and operation groups at follow-up although both reported other health benefits for the surgical group compared with the watchful waiting group: 0'Dwyer et al.: 10 more positive change in health; Fitzgibbons et al.:9
Correspondence: Rachael Powell, School of Psychological Sciences, University of Manchester, Coupland 1 Building, Oxford Road, Manchester M13 9PL, UK.

Tel. +44.161.2752569.

E-mail: rachael.powell@manchester.ac.uk

Key words: pain, inguinal hernia, surgery, activity limitations, patient satisfaction.

Acknowledgements: we are grateful to the consultant surgeons and secretarial staff at Aberdeen Royal Infirmary for their support. We would like to thank Professor W Alastair Chambers, Professor Marie Johnston, Professor Zygmunt Krukowski and Professor W Cairns Smith for their support for the project. Thanks also to Dr Rachel Shaw for comments on a manuscript draft.

Funding: this research was funded by a research fellowship to RP from the Chief Scientist Office of the Scottish Government Health Directorates. JB was funded by an MRC Special Training Fellowship.

Contributions: RP led the development of the research design, conducted all interviews, led the analysis of the data, drafted the paper and collated co-authors comments. LMcK advised on the development of the interview schedule, interview methodology and the data analysis process. LMcK carried out a secondary analysis of the data; findings were discussed and compared iteratively with RP's analysis to ensure reliability. PMK provided invaluable advice on the design, conduct, and writing of the study from his perspective as a consultant surgeon. JB has expertise in the study of chronic post-surgical pain and advised on the development of the study from this perspective, providing substantial input in developing the study design and contributing to paper drafts.

Conflict of interests: the authors declare no potential conflict of interests.

Received for publication: 5 November 2012.

Revision received: 16 January 2013

Accepted for publication: 16 January 2013

This work is licensed under a Creative Commons Attribution NonCommercial 3.0 License (CC BYNC 3.0).

(C) Copyright R. Powell et al., 2013

Licensee PAGEPress, Italy

Health Psychology Research 2013; 1:e18

doi:10.4082/hpr.2013.e18

greater reduction in pain unpleasantness and greater improvement in activity performance. From similar findings the authors reached different conclusions: a strategy of watchful waiting is a safe and acceptable option, ${ }^{9}$ and repair does not affect the rate of long-term chronic pain and may be beneficial to patients in improving overall health and reducing potentially serious morbidity. 10 Only Fitzgibbons et al. ${ }^{9}$ addressed 
patient satisfaction: more than $97 \%$ of participants in both groups reported being satisfied or very satisfied with their care. In the absence of clear evidence for the most appropriate treatment for people with asymptomatic inguinal hernias, it is important to understand the patient's perspective.

CPSP negatively affects daily activities.11 A fear-avoidance model proposes that negative thoughts about pain lead to pain-related fear, resulting in reduced activity where activity is associated with pain; ${ }^{12}$ activity reduction leads to a lowered threshold for experiencing future pain due to disuse and reduced muscle strength. 13 Evidence supporting this model is reviewed by Leeuw et al. ${ }^{14}$ It has also been demonstrated that Johnston's, 15 model incorporating Theory of Planned Behaviour cognitive constructs into the World Health Organisation's models of disability16-18 accounts for more variance in activity limitations in people with pain than pain severity alone. ${ }^{19}$ In the case of CPSP, while pain may affect activity, pain-related fear and beliefs (e.g. that limiting activity minimises damage to the surgery site) could also be important.

The present study aimed to explore experiences and understandings of pain and activity limitations as people progress along the surgery pathway. Of particular interest was whether experiences of pain and activity limitations would affect satisfaction with the decision to have surgery. Qualitative interviews were conducted to gain an in-depth understanding of the process of undergoing inguinal hernia surgery from the patient's perspective.

\section{Materials and Methods}

This study was approved by Grampian Research Ethics Committee and NHS Grampian Research and Development.

\section{Design}

Two participant groups were included: the Longitudinal and Single Interview groups. Longitudinal group interviews were conducted at three time points: two weeks before surgery (T1) and two weeks (T2) and four months postsurgery (T3). These were important timepoints: T1, surgery was imminent with a confirmed appointment; T2, the participant was recovering post-operatively; $\mathrm{T} 3$, residual postoperative pain or activity limitations were apparent. To ensure we included participants with CPSP, a second group of people who reported pain at 4 months post-surgery were recruited and interviewed once (Single Interview group). Twenty-nine interviews were conducted in total.

\section{Participants \\ Longitudinal group}

Seven adult males scheduled for elective inguinal hernia repair surgery at a hospital in Northern Scotland participated. Medical secretaries informed people of surgery dates by post approximately two weeks pre-operatively. On sending this letter, secretaries also sent a study pack containing study information. Twenty-eight packs were sent; seven people responded positively and constitute our sample. Interviews were conducted between May 2006 and January 2007.

\section{Single Interview group}

Participants were ten adult males identified as experiencing pain 4 months post-operatively from a larger, quantitative study. ${ }^{20,21}$ Participants had surgery between January and August 2007; interviews occurred between June 2007 and January 2008. Male gender was not an inclusion criterion; the sample reflects the gender distribution for inguinal hernia. ${ }^{1}$

\section{Information about chronic \\ post-surgical pain}

As part of the study's informed consent procedure, all participants received an information sheet which mentioned that some participants experience CPSP - Most hernia surgery patients do very well after hernia surgery but some patients suffer pain or discomfort that lasts longer than the usual healing time. At the time of the study, hospital leaflets regarding minor surgery did not mention the risk of CPSP. Prior to surgery, information was provided to patients by their surgeon or another member of the surgical team at an outpatient consultation. Final written consent was obtained on the day of surgery by a member of the surgical team. It is possible that some surgeons communicated the risk of CPSP to patients but there was no standardised approach to this.

\section{Data collection}

The interview began in an open-ended manner, inviting participants to describe everything that happened from when they first suspected a problem (pre-surgery interview) or from when they underwent surgery (postsurgery interviews). The topic guide comprised open-ended questions including: understanding and perception of pain; pain coping strategies; pain control; activity limitations; expectations about activity and pain control; reasons for deciding to have surgery; comparisons between time periods (pre- and postoperative pain and activity levels); perceived causes of the hernia and satisfaction with the decision to have surgery. Participants were interviewed at the University when possible; other interviews occurred in the participant's home, workplace, or (one interview) at the hospital. Interviews were tape-recorded and lasted approximately one hour.

\section{Analysis}

Transcribed interviews were analysed thematically and the participant's perception of events was taken as central. Analysis was interpretative, recognising the interaction between researcher and data. Analysis was performed manually using a Framework approach. 22 Transcripts were read and re-read; thoughts, comments and emerging themes were noted on the transcript. A list of superordinate and sub-themes was constructed. Transcripts were coded so that charts indexing extracts belonging to each theme for each participant could be produced without losing sight of how extracts were embedded within the data. The charts enabled the results to be structured according to theme with supporting evidence given verbatim. Detailed analysis records were kept by the first author and were discussed and compared iteratively with the analysis of a co-author (LMcK). Analysis was grounded in the data and so not structured according to theoretical models, but discussion of the relevance of key theories is incorporated into the Discussion section. The data are integrated into the theoretical frameworks, augmenting the understanding of the data.

\section{Results}

\section{Sample characteristics \\ Longitudinal group}

Participants were aged 34 to 77 years. They underwent: laparoscopic surgery (L1 and L3), open surgery (L2), bilateral laparoscopic and bilateral open surgery (L4 and L5 respectively) and open surgery for recurrent hernias (L6 and L7). [ $L$ indicates Longitudinal group member. $S$ indicates Single Interview group member]. Participants had a range of occupations involving varying degrees of physical activity: engineer (deskwork), crane-operator, shipping engineer (some manual work), buildings inspector, parttime security work (deskwork/walking), teacher (deskwork/walking/standing); one participant was retired. L3's response to the study invitation was received after his surgery so he was first interviewed after surgery but questions about pre-surgical experiences were asked retrospectively at T2. L6 was too ill with another condition to undertake the T3 interview. T1 interviews occurred between 1 and 15 days before surgery (median: 13.5 days); T2 interviews from 7 to 16 days post-surgery (median: 14 days); T3 interviews from 18 to 23 weeks post-surgery (median: 19 weeks). 


\section{Single interview group}

Participants were aged 34 to 69 years. All underwent repair of primary, unilateral hernias. Two participants had laparoscopic surgery (S1 and S10); all others had open surgery. Five participants were retired and one participant was his wife's caregiver. The remaining participants were: delivery worker, medical professional, safety inspector (some physical work), taxi driver and an oil industry worker (deskwork). The median time between surgery and the interview was 22 weeks (range: 20 to 30 weeks).

\section{Pain}

\section{Pre-surgery}

Most participants in both groups reported some pre-operative discomfort but this tended to be characterised as bearable: uncomfortable when coughing, not really, no pain [L7(T1)]; just an ache (S2). Exceptions included S9 for whom the pain was more intense: it was getting uncomfortable, seriously uncomfortable. Participants did not seem unduly concerned about the discomfort; they appeared stoical or pragmatic and were prepared to tolerate some pain: just put up with it [L3(T2) retrospectively].

\section{Two weeks post-surgery}

All participants experienced postoperative pain although some Longitudinal participants already reported little or no current pain. Pain was often considered a natural consequence of surgery and therefore not a cause for concern: they've sliced you open so you're going to feel pain (S4). Worry arose when pain differed to expectations: it's when you can see parts you didn't have that's nothing to do with the operation, why is it so painful there? [L5(T2)]. This participant experienced pain and bruising in the scrotum. Another man was concerned because his pain was similar to that experienced before surgery; he worried that the repair was unsuccessful: it's the same, same sort of thing I feel now, which doesn't give you much confidence [L1(T2)]. Thus, the pain itself did not cause concern, but interpreting the pain as indicating a possible problem led to worry.

Some Single Interview participants talked about pain as an indicator of what they could do without causing damage: it would be down to the fact that yeah well I shouldn't be doing that right now (S2). One participant reported being afraid to move because of the pain: Frightened to move, frightened to cough, frightened to breathe, frightened to sneeze (S4). If a fear-avoidance model of pain is applied to the context of CPSP it would be expected that participants who experienced CPSP (all Single Interview participants) would be likely to report pain-related fear or anxiety. This was not so: S4 was unusual in reporting pain- induced fear. Nevertheless, Single Interview participants' recall of pain-related emotions in these retrospective interviews may not accurately reflect their feelings at the time.

\section{Four months post-surgery}

Most Longitudinal group participants reported no residual sensations. L4 reported sometimes feeling pressure. L5 and L7 experienced occasional twinges but neither expressed concern about this. L5 seemed to interpret the pain positively as part of the healing process: it's a healing up thing you know when it's knitting [L5(T3)]. This excerpt continues the concept of interpreting pain within the context of a natural healing time and process. It appears that if the sensation indicated a stronger, healthier future it was not perceived as problematic. However, there was evidence of reappraisal for L5: For a while before, about two, three weeks ago when I was getting more of them I was thinking, God is this what you're talking about am I going to have this for the rest of my life?.

The extent to which this participant was concerned seemed to be associated with his pain experience - as the frequency of twinges reduced, instead of perceiving the pain to be a never-changing condition, he started viewing it as something that was part of the healing process. This participant had catastrophized about the pain (showing an exaggerated negative orientation toward noxious stimuli). ${ }^{23}$ Catastrophizing has predicted acute pain after surgery but, ${ }^{24}$ for this participant, the frequency of twinges reduced over time, leading to reassurance. The catastrophizing in this instance may have been intensified by our research. The process of informed consent made clear that we were investigating postsurgical pain which some may not have otherwise worried about.

All Single Interview participants reported pain four months post-surgery. However, for some, the pain was described as having minimal impact. Others experienced more frequent pain which appeared not to greatly affect them; words used seemed to downplay the pain, e.g.: not serious but still there...more of an irritant than a pain (S1). Making sense of the pain was an important theme. Some participants accepted pain as a natural by-product of surgery: you cannae have prods in your stomach and get away Scot free (S2). S1 questioned the surgical approach, wondering about alternatives, and felt responsible for not seeking clarification about potential consequences and treatment choices: I wonder about the best surgical policy... whether you have to have keyhole or you have to have a more conventional incision...I think I should have been asking more questions. Others seeking causes for their pain looked to their own behaviour: some attributed the cause to being too active too soon: the thought that you've damaged something... because you've done something, too much too early (S6). Unlike L5, these participants seem not to have considered the risk of CPSP, despite being presented with similar information. Instead, it seems that the idea of surgery as a fix persisted; they looked to other causes for their pain.

Attributing the pain to causes other than the healing process could lead to concern. For S8, the pain was central to a range of concerns: whether the operation had been effective and whether the pain might result from another health problem. Thus, it was the perception that the pain resulted from something more serious that caused concern, not the pain per se. In contrast, concern was minimised if pain was viewed as part of the surgery process: it's part and parcel of having that operation...I know what the ache is all about so I don't really concern myself (S2).

These accounts suggest there is considerable diversity in how pain is experienced. Healing pain was considered a positive concept that was used to modify the experience of pain. Other pain was taken as a portent of underlying serious damage. All participants sought to interpret their pain and assess what was within normal parameters.

\section{Activity limitations}

\section{Pre-surgery}

Participants mostly continued with normal activities before surgery but many reported limiting certain behaviours e.g. lifting. Predominantly, the reason given for limiting activity was avoidance of further damage to the hernia site, not pain avoidance: there's a little hole there now and if I go berserk there's going to be a big hole (S4). Participants may have found it easier to admit to restricting activity to avoid damage than because of pain but the activities limited were not necessarily painrelated: I avoid some of the heavier lifting. But actually I think standing is more uncomfortable (L2(T1)). In one case caution was triggered on diagnosis: the doctor said oh it's a hernia and he explained what the hernia was, from then on ... I was more cautious of the way I lifted (S4). Few participants reported limiting activity pre-operatively because of fear of pain. However, pain experience was reported as a signal to reduce activity by some Single Interview participants: if it was painful I stopped and rested (S10).

\section{Two weeks post-surgery}

Most participants in both groups reported limiting activity. While limiting pain was reported as one motive, a key intention was to avoid damage, to let the wound heal: I was concerned that I was going to open the wound 
again with too much activity (S1). Participants used pain as an indicator that an activity was too much and damage was risked: that was a big warning, you can't do that [L5(T2)]. Limiting activity may have fulfilled the dual purposes of minimising damage and minimising pain; S7 discussed how pain reduced his activities but also reported being careful because after previous (non-hernia) surgery he had been active sooner than advised and it [the wound] burst.

\section{Four months post-surgery}

In the Longitudinal group, some participants reported limiting activity despite experiencing little or no pain to reduce the risk of hernia recurrence. For example, L4(T3) was avoiding heavy lifting because he did not perceive the hernia site to be strong enough: it's still a weakness there like you know. This participant did not report pain but he perceived the healing process to be on-going. His expectations from before surgery were that it was important to wait for the repair site to become strong: when she'd [the surgical registrar] explained to me what was happening...you realise well I can't go toddling back off-shore and start doing things like that. Thus, L4 appeared to understand healing as a slow, complex process, and from an early stage had no expectation that usual activity would resume quickly. Activity avoidance was a rational, cognitive response to a threat, rather than a response to fear of pain.

The understanding of what the surgery process had involved also impacted upon L5's perception of healing: I am still thinking I've got to be careful because I don't want them reappearing, I don't know how far that net that they put in moves and I think well I don't want things appearing round the sides of the nets and all the rest of it. There is still this in your mind but as far as pain wise it's just life goes on and you can't stop for it. L5 perceived the mesh used by surgeons to be insecure, with the potential for the hernia to escape and reemerge. While pain was disregarded as an indicator to reduce activity, the image of the hernia emerging led to caution.

Most Single Interview participants reported limiting activity to some extent. Avoiding recurrence or damage to the site was, again, an important goal: if I had a hernia on the other side... or damaged the original one, I'd feel pretty angry with myself... so I am cautious (S6). Reducing activity was not necessarily considered a limitation - it could be perceived as a positive lifestyle change. S4 felt the hernia surgery had no effect on what he achieved, but he modified his approach. Lifting was important in his job and he reduced the number of items carried simultaneously rather than avoid lifting altogether: So in that respect
I am limited but it's a good limitation. This response demonstrates that participants may differ in how they understand questions about activity limitations. This participant focussed less on how activities were completed and more on what was achieved - as the same task was accomplished, the change was not regarded as a limitation. Instead, the change was viewed as a benefit as his current behaviour could protect him from future problems.

The findings here suggest many participants had a strong sense of wound site vulnerability and activity was calibrated in a way that is protective. Activity limitation purely to avoid pain was not dominant.

\section{Satisfaction with the decision to have surgery}

At the four-month interviews, participants compared their pain and activity levels with those before surgery. Longitudinal participants reported no change or an improvement in pain and activity and seemed highly satisfied with the decision to have surgery. For some, this satisfaction seemed to be connected with improved functioning or reduced pain intensity. Many were also pleased to have prevented future problems and anxiety regarding complications: it was just once I found out what could, what could happen well that was really what I wanted the operation for...I'm not so worried about them now [L5(T3)]. When Longitudinal Group participants were asked, pre-operatively, why they chose to undergo surgery, none mentioned pain reduction, tending to focus on the threat of complications. The concept of surgery as a reliable fix for a malfunctioning system was voiced: It is there so I want it put right [L6(T1)]. This participant did not seem worried either about pain or about potential long-term consequences, but instead viewed the body as a machine where if something goes wrong, it should be mended.

Single Interview participants reported being at least as active as they were before surgery, and having similar or lower pain levels than before surgery. However, some experienced worse pain at four months than before surgery: When I went for the surgery I really was in no pain... where I am just now is that I'm still in pain (S8). Nevertheless, no one reported dissatisfaction with the decision to undergo surgery. For example, even though S8's recovery had been disappointing, he still believed he had made the right decision: I would have rather had the surgery than run the, that risk. It would seem that, despite experiencing persisting pain, participants were happy with their decision to undergo surgery because their goals had been met. The benefits from surgery were perceived as reducing the risk of future complications or of the hernia worsening: it was going to progress (S5), fixing a problem: because once you're told that something's nae right...get it done (S7) and maintaining a good level of activity: had I not got the operation, then I would have certainly slowed down my activities (S2). These perceived benefits tended to reflect reasons given for having surgery e.g. reducing the risk of complications and fixing a problem. S10 thought that an operation was unavoidable: you've got to have surgery for a hernia. In the context of discussing pre-surgical pain, one participant did report that the surgery was important because of his pain and yet, when asked more directly about reasons for having surgery, cited a desire to return to his more active pre-hernia lifestyle: it needed to be done, because it was getting uncomfortable (S9, context: discussing pain level); fix it so that I could get on with doing some of the other things that I used to do whether it be golf or tennis or whatever (S9, context: reasons for surgery). It is not clear whether return to activity was a more salient reason for having surgery, or whether it was easier for him to give activity as a primary reason than to admit that the discomfort was bothering him enough to make him seek a fix.

In summary, even when four-month pain was more severe than pre-operative pain, participants were happy with their decision to have surgery. It appears that surgery enabled them to achieve their aims, avoiding the perceived risk of more serious health problems. Surgery was conceived of as providing both an immediate fix and a guarantee against complications.

\section{Discussion}

All the participants interviewed in this study expressed satisfaction with their decision to have surgery, even when pain and/or activity limitations were experienced four months later. However, severe, continuous pain at four months was rare. Participants tended not to catastrophize about their pain; pain or discomfort rarely caused concern if it was perceived as part of the usual process. Before surgery, many participants reported some discomfort but pain alone did not appear to restrict activity; restricted activity was tied to concern about damage to the hernia site. Immediately after surgery, the risk of damage to the wound was a key incentive in limiting activity. At four months, pain and activity appeared to have a degree of independence with some pain-free participants limiting activity (to avoid wound damage).

The association between pain and activity limitation is not necessarily direct. Dixon et al. ${ }^{19}$ demonstrated that psychological factors (control beliefs) mediated the relationship between pain and activity limitations in people awaiting joint replacement surgery. In the present study, some participants interpreted 
pain as a sign of continued physical weakness and limited activity to avoid aggravating the problem. Even without pain, the wound site could be perceived as vulnerable and protective steps were taken: activity was restricted. While pain-related fear sometimes seemed important, it was not necessary for participants to be fearful of pain to limit activity. Fear-avoidance models 12 may be more relevant to CPSP in the longer term than at four months post-surgery when pain may still be attributed to the normal recovery process and the need to prevent damage to the hernia site is salient.

A striking finding was that no participant reported dissatisfaction with the decision to have surgery, even when they experienced CPSP. The Common Sense Self-Regulation Model is helpful in understanding these findings. ${ }^{25,26}$ It proposes that, when faced with a health threat, we form representations to understand the threat in terms of its identity, timeline, cause, consequences and control. Decisions about coping actions are taken according to these appraisals. A key reason for having surgery was to avoid potentially serious consequences such as strangulation or obstruction. Awareness of consequences was accompanied by a belief that surgery would control or fix the problem. Thus, surgery seemed to be internalised as an appropriate coping action in dealing with the perceived drastic consequences of leaving the hernia untreated.

We did not ask participants to what extent they felt at risk of negative events resulting from the hernia but it is likely their perception of risk was higher than the actual risk reported. ${ }^{8-10}$ At the extreme, one participant believed there was no alternative to surgery. Future research should address the likelihood of choosing surgery (rather than watchful waiting) at different levels of perceived risk of serious adverse events, including risk of CPSP. Fitzgibbons et al. found that over $97 \%$ of participants in both surgery and watchful waiting groups reported being satisfied with their care. ${ }^{9}$ Being given information about the option of watchful waiting in the study consent procedure may have reassured participants about the risk of obstruction or strangulation. Powell et al. found that information and advice provided by health care professionals was taken very seriously by participants (a subgroup of the present sample) when making decisions regarding surgery and recovery. 27 The participants of the present study were referred to surgical clinics by their family doctors, and all opted for surgery. After referral, a surgical team is responsible for the person's care so it could be expected that the focus of professionals and patients will be on surgical management. Where conservative management (watchful waiting) is presented alongside surgery, people may be more open to alter- native management approaches. It would be useful to investigate reasons for treatment choices in people who are not referred to a surgical team, or who decide not to undergo surgery after discussion with the surgical team.

This study explored factors related to the decision to have hernia surgery, satisfaction with surgery and return to activity. However, the extent to which the results can be generalised is limited by the size and representativeness of the sample. In particular, while all Single Interview participants experienced pain at four months postoperatively, this pain was mostly mild and intermittent rather than severe. Satisfaction with surgery may be lower in people with severe CPSP. A second factor that may have influenced the high reports of satisfaction is that, even though the interviewer (RP) was independent of the surgical team and assured participants that their participation was confidential, the separation of research from surgical care may not have been understood by participants because, following standard ethical guidelines, participants were recruited using a letter from a surgeon at their hospital.

We were unable to conduct a longer term follow up with these participants, e.g. to 1 year after surgery, because of practical constraints on the project timeline. However, as the CPSP experienced at 4 months after surgery was already generally low, the benefits of having a longer follow-up may be limited with our particular sample of patients.

A possible unintended impact of the study was that the information provided about CPSP as part of the study information may have raised awareness about CPSP. Caution is needed here as the causal link is inferred. As negative emotions can increase pain perceptions and predict higher post-operative pain, ${ }^{28,29}$ this could have negative consequences for pain experience. Conversely, given that this study found pain to cause concern when it differed from expectation, understanding that CPSP may occur could be reassuring. This observation clearly raises ethical implications and researchers need to be mindful of this issue. Ideally, participants would be fully informed about the risks of surgery (including CPSP) by the care team so information provided by a study such as this should not introduce risk information of which patients are unaware.

\section{Conclusions}

While some participants experienced CPSP after hernia surgery, it did not cause undue concern for most and dissociation between pain intensity and activity limitation was observed: many participants primarily limited activity to avoid damage to the wound site rather than to avoid pain. Importantly, participants reported being satisfied with the decision to have surgery even when pain persisted postoperatively because they believed that surgery had averted the risk of dangerous complications.

These findings have significant implications for patient care. First, further research is needed to ascertain whether patients, particularly those with pain-free hernias, are fully aware of the actual risk of serious consequences when surgery is delayed or not pursued, and to address how this understanding impacts on deciding whether or not to undergo surgery.

Second, it is important to ensure that patients are fully informed of all risks associated with the surgical procedure, including the risk of CPSP. In response to the present study and other research which has increased local awareness of CPSP,3,21 a website was developed for patients in the Grampian Region which is now used to guide the consent process and covers risks of surgery including CPSP. Further research is needed to assess the impact of providing such additional information on uptake of surgery and patients' experience of the surgical and recovery processes.

\section{References}

1. Wilson N. Inguinal hernia. In: Lattimer CR, Wilson NM, Lagottolla NRF, eds. Key topics in general surgery. 2nd ed. Oxford: BIOS Scientific Publishers Ltd; 2002. pp 179-81.

2. International Association for the Study of Pain Subcommittee on Taxonomy. Classification of chronic pain. Descriptions of chronic pain syndromes and definitions of pain terms. Pain. 1986;24 Suppl 1:S1-S226.

3. Poobalan AS, Bruce J, Smith WCS, et al. A review of chronic pain after inguinal herniorrhaphy. Clin J Pain 2003;19:48-54.

4. Bay-Nielsen M, Perkins F, Kehlet H. Pain and functional impairment 1 year after inguinal herniorrhaphy: a nationwide questionnaire study. Ann Surg 2001;233:1-7.

5. Paily A, Thornton M. Chronic pain following a Lichtenstein inguinal hernia repair: a clinical and legal dilemma. ANZ J Surg 2009;79:517-20.

6. National Institute for Clinical Excellence. Laparoscopic surgery for inguinal hernia repair. London: National Institute for Clinical Excellence: 2004.

7. Hair A, Paterson C, Wright D, et al. What effect does the duration of an inguinal hernia have on patient symptoms? J Am Coll Surg 2001;193:125-9.

8. Leubner KD, Chop WM, Ewigman B, Loven B. What is the risk of bowel strangulation in an adult with an untreated inguinal her- 
nia? J Fam Pract 2007;56:1039-41.

9. Fitzgibbons RJ, Giobbie-Hurder A, Gibbs J0, et al. Watchful waiting vs repair of inguinal hernia in minimally symptomatic men. JAMA 2006;295:285-92.

10. O'Dwyer PJ, Norrie J, Alani A, et al. Observation or operation for patients with an asymptomatic inguinal hernia. Ann Surg 2006;244:167-73.

11. Courtney CA, Duffy K, Serpell MG, O'Dwyer PJ. Outcome of patients with severe chronic pain following repair of groin hernia. Br J Surg 2002;89:1310-4.

12. Vlaeyen JWS, Linton SJ. Fear-avoidance and its consequences in chronic musculoskeletal pain: a state of the art. Pain 2000;85:317-32.

13. Bortz WM. The disuse syndrome. West J Med 1984;141:691-4.

14. Leeuw M, Goossens MEJB, Linton SJ, et al. The fear-avoidance model of musculoskeletal pain: current state of scientific evidence. J Behav Med 2007;30:77-94.

15. Johnston M. Models of disability. Psychologist 1996;9:205-10.

16. Ajzen I. The theory of planned behavior. Organ Behav Hum Decis Process 1991;50:179-211.

17. World Health Organisation. International classification of functioning, disability and health. Short version. Geneva: World Health Organisation; 2001.

18. World Health Organisation. International classification of impairments, disabilities and handicaps. Geneva: World Health Organisation; 1980.

19. Dixon D, Johnston M, Rowley D, Pollard B. Using the ICF and psychological models of behavior to predict mobility limitations. Rehabil Psychol 2008;53:191-200.

20. Powell R, Johnston M, Bruce J, et al. Psychological predictors of chronic pain after hernia surgery and the impact of chronic pain on activity limitations and quality of life. Final report of Research Training Fellowship to the CSO, December 2008. CSO reference number: CZF/01/33.

21. Powell R, Johnston M, Smith WCS, et al. Psychological risk factors for chronic postsurgical pain after inguinal hernia repair surgery. Eur J Pain 2012;16:600-10.

22. Ritchie R, Spencer L. Qualitative data analysis for applied policy research. In: Bryman A, Burgess RG, eds. Analysing qualitative data. London: Routledge; 1994. pp 173-94.

23. Sullivan MJL, Bishop SR, Pivik J. The pain catastrophizing scale: development and validation. Psychol Assess 1995;7:524-32.

24. Sullivan M, Tanzer M, Stanish W, et al.
Psychological determinants of problematic outcomes following total knee arthroplasty. Pain 2009;143:123-9.

25. Leventhal H, Nerenz DR, Steele DJ. Illness representations and coping with health threats. In: Baum A, Taylor SE, Singer JE, eds. Handbook of psychology and health. Hillsdale: Lawrence Erlbaum Associates, Inc; 1984. pp 219-52.

26. Leventhal H, Brissette I, Leventhal EA. The common-sense model of self-regulation of health and illness. In: Cameron LD, Leventhal $\mathrm{H}$, eds. The self-regulation of health and illness behavior. London: Routledge; 2003. pp 42-65.

27. Powell R, McKee L, Bruce J. Information and behavioural instruction along the healthcare pathway: the perspective of people undergoing hernia repair surgery and the role of formal and informal information sources. Health Expect 2009;12: 149-59.

28. Rainville P, Bao QVH, Chrétien P. Painrelated emotions modulate experimental pain perception. Pain 2005;118:306-18.

29. Munafò MR, Stevenson J. Anxiety and surgical recovery - Reinterpreting the literature. J Psychosom Res 2001;51:589-96. 